\title{
Identification of Immune-related IncRNA Panel for Predicting Immune Checkpoint Blockade and Prognosis in Head and Neck Squamous Cell Carcinoma
}

\section{Chongchang Zhou}

Ningbo Medical Center Lihuili Hospital

Guowen Zhan

Ningbo Yinzhou Second Hospital

\section{Zhisen Shen}

Ningbo Medical Center Lihuili Hospital

\section{Yi Shen}

Ningbo Medical Center Lihuili Hospital

Hongxia Deng

Ningbo Medical Center Lihuili Hospital

\section{Yiming Shen}

Ningbo Medical Center Lihuili Hospital

Jianing Wang

Ningbo Medical Center Lihuili Hospital

Jinyun Li ( $\nabla$ fylijinyun@nbu.edu.cn )

the Affiliated Hospital of Medical School of Ningbo University

\section{Research Article}

Keywords: head and neck squamous cell carcinoma, immune-related long non-coding RNAs, immunity cell infiltration, immunotherapy, drug sensitivity

Posted Date: September 3rd, 2021

DOI: https://doi.org/10.21203/rs.3.rs-858241/v1

License: (c) (1) This work is licensed under a Creative Commons Attribution 4.0 International License. Read Full License 


\section{Abstract}

Immunotherapy is changing head and neck squamous cell carcinoma (HNSCC) treatment pattern. According to the Chinese Society of Clinical Oncology (CSCO) guidelines, immunotherapy has been deemed as first-line recommendation for recurrent/metastatic HNSCC, marking that advanced HNSCC has officially entered the era of immunotherapy. Long non-coding RNAs impact every step of cancer immunity. Therefore, reliable immune-IncRNA able to accurately predict the immune landscape and survival of HNSCC are crucial to clinical management. In the current study, we downloaded the transcriptomic and clinical data of HNSCC from The Cancer Genome Altas and identified differentially expressed immune-related IncRNAs (DEir-IncRNAs). Further then, Cox and least absolute shrinkage and selection operator (LASSO) regression analyses were performed to identify proper DEir-IncRNAs to construct optimal risk model. Low-risk and high-risk groups were classified based on the optimal cut-off value generated by the areas under curve for receiver operating characteristic curves (AUC), and KaplanMeier survival curves were utilized to validate the prediction model. We then evaluated the model based on the clinical factors, immune cell infiltration, chemotherapeutic and immunotherapeutic efficacy between two groups. Our results constructed a risk model consisted of 18 DEir-IncRNA pairs showing significantly association with survival of patients with HNSCC. Besides, HNSCC patients with low risk score significantly enriched of $\mathrm{CD} 8^{+} \mathrm{T}$ cell, and corelated with high chemosensitivity and immunotherapeutic sensitivity. In summary, our risk model could be served as a promising clinical prediction indicator, effective discoursing of the immune cell infiltration of HNSCC patients, and distinguishing patients who could benefit from chemotherapy and immunotherapy.

\section{Introduction}

Head and neck cancer mainly arise in the oral cavity, oropharynx, hypopharynx, and larynx, and head and neck squamous cell carcinoma (HNSCC) is the predominant histology. HNSCC was the seventh most common cancer worldwide ${ }^{1}$. Multiple pathogenic factors (including heavy use of tobacco, alcohol and chronic infection of human papilloma virus) have been proven in association with the initial of HNSCC ${ }^{2,3}$. Smoking could increase the risk of HNSCC 5 to 25 -fold and the risk increase with the quantity of cigarette ${ }^{3,4}$. The consumption of alcohol also independently doubles the risk of HNSCC $^{5}$.

Cancer is characterized by the accumulation of various of genetic alterations, which results in the acquisition of six biological capabilities during the multistep development of human tumors ${ }^{6}$. Subsequent molecular studies revealed that these genetic or epigenetic alterations often product mutated cellular antibodies or biomarkers, aberrantly expressed normal genes ${ }^{7-9}$. These abnormal expressed molecules on the surface of cancer cells could be recognized by $\mathrm{CD} 8^{+} \mathrm{T}$ cell, distinguishing them from their normal counterparts and even inhibiting the outgrowth of cancer cells ${ }^{10}$. Therefore, in the past decades, researchers dedicated to efficiently activate cancer immunity system to fight cancer cells, which is called "immunotherapy". However, the cancer immunity cycle does not perform optimally in cancer patient; sometimes, rarely protective efforts, even promoting assists by $\mathrm{T}$ cell were found during 
the progression of human cancers ${ }^{11-13}$. Either tumor antibodies may not be detectable, or dendritic cells and $T$ cells may classify these antigens as self thereby creating low ratio of cytotoxic $T$ lymphocytes ${ }^{14-16}$. Besides, negative regulators to $T$ cell responses are also responsible for the failure of immune protection ${ }^{17}$.

The goal of cancer immunotherapy is to initiate or reinstate the cycle of cancer immunity to exert antitumor effects. Therefore, the cancer immunotherapies must be carefully configured to overcome these negative feedback mechanisms. Indeed, immunotherapy exhibits marvelous efficacy in clinical trials ${ }^{18}$; however, only a minority of patients have incredible responses to immunotherapy in clinical practice. Therefore, a deep understanding of immune system and identification of these sort of HNSCC patients will be helpful to develop effective therapeutic strategies for cancer immunotherapy and to improve the overall survival of HNSCC.

The pro-tumorigenic or anti-tumorigenic effects of cancer immune system exerted is rely on specific microenvironment ${ }^{19,20}$ Numerous factors in the tumor microenvironment can act to modulate the existing activated antitumor T cell immune response, such as expression of PD-L1 or PD-1 ${ }^{21,22}$. Long non-coding RNAs (IncRNAs) are a series of RNAs without protein-coding capacity ${ }^{23}$, which were once considered as "garbage sequences". However, increasing evidence has shown that the crucial roles of IncRNAs in a variety of cellular and biological processes ${ }^{24}$. There are numerous abnormally expressed IncRNAs associated with cancers initiation and metastasis ${ }^{25-27}$. In prostate cancer, several IncRNAs were highly specific to prostate cancer, including prostate cancer antigen 3 (PCA3), prostate cancer gene expression marker 1 (PCGEM1), and prostate cancer-associated transcript 1 (PCAT-1) ${ }^{28-30}$. HOTAIR (HOX transcript antisense intergenic RNA) is one of the most well-studied IncRNAs that is overexpressed in a variety of cancers including breast, colorectal, hepatocellular, gastrointestinal, and non-small cell lung carcinomas 31-35. Besides, abundant evidence showed that abnormal expression of IncRNAs also impact every step of cancer immunity ${ }^{36,37}$. The two main classes of the immune system are the innate immune system and the adaptive immune system ${ }^{38}$. They are both participated in eliminating cancer cells. The innate immune system is the first line of immunity, including macrophages, Natural killer (NK) cells, and neutrophils. According to the polarization state of macrophages, they can be divided into classically activated macrophage (M1) and activated macrophage (M2) phenotypes. M1 have anti-tumor properties, while M2 have tumor-promoting abilities ${ }^{39}$. TCONS_00019715 might play a critical role in promoting macrophage polarization to the M1 phenotype, which enhances tumoricidal activities ${ }^{40}$. LncRNA-cox- $2^{2}$ had a higher expression in M1 macrophages than M2 macrophages ${ }^{41}$. NK cells as a critical component of innate immunity are crucial to mediate antitumor immune responses. Increasing evidence showed that IncRNAs can also be involved in regulating NK cell cytotoxicity ${ }^{42-45}$. Down-regulated expression of IncRNA GAS5 were observed in liver cancer and promoted tumor progression by inhibiting NK cell activity, while exogenously increasing expression of IncRNA GAS5 could elevated NK cell cytotoxicity ${ }^{42}$. In cervical cancer patients, the cytotoxic activity of NK cell is facilitated by the expression of LINC00240 45 . 
These studies highlighted the potential roles of IncRNA in regulating cancer immunity. A better understanding of IncRNA-mediated regulation of cancer immunity will provide novel targets for the development of new therapeutic strategies in HNSCC. In the present study, we aim to comprehensively explore immune-related IncRNA expression profile and construct a model to prognosis outcome, drug sensitivity, and implement clinical strategies in HNSCC.

\section{Results}

\section{Acquisition of differential immune-related IncRNAs}

We firstly abstracted the IncRNA and mRNA matrix from transcriptome RNA-sequencing data of The Cancer Genome Altas (TCGA, https://www.cancer.gov/about-nci/organization/ccg/research/structuralgenomics/tcga). Subsequently, the intersection, namely immune-related mRNAs (ir-mRNAs) of TCGA, achieved between mRNA matrix of TCGA and a list of well-known immune-related genes from ImmPort database. Then, the immune-related IncRNAs (ir-IncRNAs) were obtained by Pearson's analysis between IncRNA matrix and ir-mRNAs of HNSCC from TCGA. Eventually, we obtained a total of 804 ir-IncRNAs. Subsequently, we identified differentially expressed ir-IncRNAs (DEir-IncRNAs) between 501 HNSCC tumor tissues and 44 normal tissues, the results showing 256 DEir-IncRNAs with FDR adjusted $P<0.05$ and absolute log fold change (FC) >1 (Table S1). These DEir-IncRNAs were represented by heatmap and volcano plots in Figure 1.

\section{Identification of survival-related DEir-IncRNAs pair}

We cyclically paired DEir-IncRNAs pairs through comparison of all the DEir-IncRNAs expression in each HNSCC patient of TCGA. Then, univaritae Cox regression was performed to identify survival-related DEirIncRNAs pairs, and 253 DEir-IncRNAs pairs were step into the flowing analyses. Taking into account the large number of confounding factors caused by multiple variables, we applied LASSO regression to obtain the best DEir-IncRNAs pairs to improve the effectiveness of subsequent analysis. Eventually, a total of 18 DEir-IncRNAs pairs were identified and applied for establishment of a risk model of HNSCC.

\section{Establishment of a risk model}

Based on the results of LASSO regression, a total of 61 DEir-IncRNA pairs was stepped into multivariate Cox proportional hazard regression analysis to specify the variables in the model formula, and 18 DEirIncRNA pairs were determined. The formula for calculation the risk score is: $0.324^{*}$ expression of (LINC01063|AL139288.1)-0.250*expression of (MIR9-3HG|AC091563.1)-0.295*expression of (MIR9-3HGIMIR924HG)

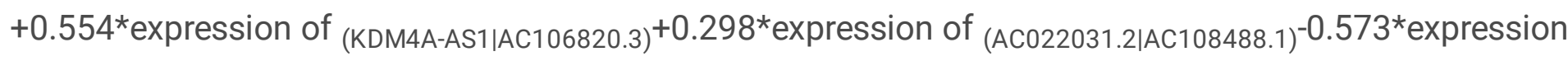

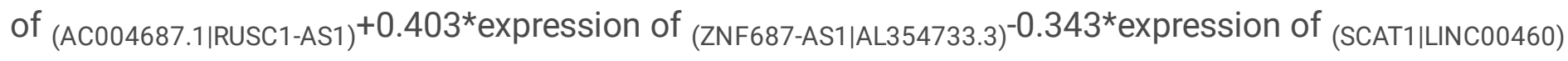
$-0.248^{\star}$ expression of (AC104825.1|KTN1-AS1) $+0.378^{\star}$ expression of (AL133243.2|AC022144.1) $0.371^{\star}$ expression of (HS1BP3-IT1|LINC02454) $+0.4102 *$ expression of (AC112484.3|C5orf66-AS1) $^{+0.420 *}$ expression of (AC132192.2|AC004148.1) $^{+0.345^{*} \text { expression of }(\text { MIR1-1HG|AC007991.2) }}+0.340^{*}$ expression of 
(AL133353.1/AC083964.1) $^{+0.380 *}$ expression of $\left(\right.$ AP003555.1|P3H2-AS1) ${ }^{-0.281 * e x p r e s s i o n ~ o f ~}$

(AC008735.2|SNHG25) ${ }^{-0.357 *}$ expression of (AC012236.1|AC060766.4). The risk score of each sample of TCGA were computed and then calculated the areas under curve for each receiver operating characteristic (ROC) curve of 18 pairs. The distribution of risk score of each sample were exhibited in Figure 2. The maximum area under curve of ROC (AUC) is 0.810 (Figure 3A), and the optimal cut-off point to differentiate the high- or low risk-group among patients with HNSCC is 1.429 (Figure 3B).

\section{Validation of the risk model}

According to the cut-off point confirmed above, 195 patients were grouped into high risk group and 304 into low risk group. Kaplan-Meier analysis showed that patients in the low-risk group exhibited a longer survival time than those in the high-risk group $(P<0.0001)$ (Figure 4). Subsequently, we re-performed stratified analysis between high and low-risk group by clinical characteristics (such as gender, age, TNM stage and grade), and subgroup analysis results robustly supported that better overall survival of patients in low-risk group than in high-risk group (Figure S1). In order to avoid basis from clinical features, univariate and multivariate Cox proportional hazard regression analysis were performed to evaluate the independent prognostic value of risk model. The results showed that our risk model, clinical stage and age could be served as independent prognostic indicator (Figure 5).

Then, we assessed the relationship between the risk score of HNSCC and clinicopathological characteristics (TNM stage, age, grade). The results showed that patients with advanced T stage more likely gathered in the high-risk group, indicating that higher scores of the 18 DEir-IncRNA pairs might be significantly associated with the progression of HNSCC (Figure S2).

\section{Immune cell abundance analysis based on risk model}

We analyzed the infiltration difference of immune cells between the low- and the high-risk group classified by the risk model. The immune infiltration estimations for TCGA expression profiles by CIBERSORT (https://cibersort.stanford.edu/) ${ }^{46}$. Then, the intersection of the infiltration estimation file and our risk score file were acquired for further Pearson's correlation analysis. The association between immune cell infiltration difference and different risk group were presented by vioplot (Figure 6).

Subsequent, we also assessed the association between several immune checkpoint inhibitors biomarkers and our risk model, and the results showed that low risk group were more likely with high expression of cytotoxic T lymphocyte-associated antigen-4 (CTLA4), and programmed cell death protein 1 (PD-1), whereas, the expression of programmed cell death ligand 1(PD-L1) showed no statistical differences (Figure 7).

\section{Prediction drug sensitivity based on risk model}

Considering the positive association between two important immune checkpoint inhibitor (ICI) biomarkers, we assess the innate sensitivity or resistance to anti-PD-1 therapy. Our results showed that 
HNSCC patients with low-risk score represented significantly higher IPS-CTLA4 and IPS-PD1 (immunophenoscore, IPS), indicating the potential of ICI application for HNSCC (Figure 8). Besides, we also identified associations between risk and the efficacy of common chemotherapeutics (including cisplatin, docetaxel, gemcitabine and paclitaxel) in HNSCC (Figure 9). The results indicated that HNSCC patients with low risk represented higher sensitivity to docetaxel and gemcitabine.

\section{Discussion}

HNSCC are a heterogeneous group of cancers originated from oral cavity, pharynx, and larynx, which are characterized by different molecular subgroups and clinical features. The primary treatment of early stage of HNSCC is radical radiotherapy or surgery, and the treatment option of advanced stage of HNSCC is radiotherapy combination with chemotherapy. However, a substantial proportion of HNSCC patients die from their disease after such treatment. Besides, these extensive surgery and high-dose chemotherapies are also mutilating for HNSCC patients.

The increasing understanding of tumor immunology has led to intense preclinical and clinical research to new therapy, called "immunotherapy". Advances in immunotherapy have brought hope, especially the favor outcome of clinical trials, such as KEYNOTE-012, KEYNOTE-141, KEYNOTE-055 and KEYNOTE040 47-51. Two anti-PD-1 antibodies (belongs to ICls), pembrolizumab and nivolumab, are approved for the treatment option of recurrent and metastatic HNSCC. Subgroup analysis showed that HNSCC patients with high expression of PD-L1 are deemed more sensitivity to above two antibodies. However, only a minority of patients have benefit from these two drugs in the clinical practice. Therefore, in order to identify a proportion of HNSCC patients susceptible of immunotherapy, researchers took advantage of advanced technology, such as targeted next-generation sequencing, and had constructed multiple relevant models or biomarkers to prediction. The most representative biomarker is tumor mutation burden (TMB) in lung cancer ${ }^{52}$. TMB is significantly associated with improved benefit among patients with nonsmall-cell lung cancer (NSCLC) treated with ICls and is an independent variable ${ }^{52}$. Therefore, to identify such reliable biomarkers or models for distinguishing this sort of patients or different risk patient subgroup, we performed a comprehensively analysis to the expression of multi-type of RNA profile in HNSCC.

We analyzed the expression profile of IncRNA in HNSCC for the following reasons: 1) the amount of ncRNAs in the whole genome is $98 \%$, which were once thought to be "garbage sequence"; 2 ) increasing evidence demonstrated the critical role of IncRNA in biological process and the association between IncRNA and human cancers; 3 ) recent studies promulgated the eye-catching roles of IncRNA in antitumor immunity. Firstly, we synthesized the mRNA profile of TCGA HNSCC and well-known ir-mRNA from public database. Subsequently, Pearson correlation analysis was performed to identify ir-IncRNAs. Finally, differentially expressed ir-IncRNAs (DEir-IncRNAs) were filtrated through "limma" package of R software, and a total of 256 DEir-IncRNAs were identified. Among these 256 DEir-IncRNAs (including LINC01614, LINC01322, and LINC00460, detail information presented in Table S1) were consist of an amount of wellstudied IncRNAs associated with human cancers, such as LINC01614 in breast cancer, lung cancer, and 
gastric cancer ${ }^{53,54}$. Changes in the composition, physical properties, and spatial conformation of the extracellular matrix (ECM) play an important role in the immune microenvironment of tumor and these changes are proven having a significant impact on drug sensitivity and patient survival ${ }^{55-57}$. LINC01322 was significantly enriched pathways related to the ECM, including ECM structural constituents, cell adhesion-molecule binding, actin binding, and glycosaminoglycan binding ${ }^{58}$.

It was simply not possible to assess all the expression level of 256 DEir-IncRNAs in clinical practice for the cost of human resource. Therefore, we firstly converted these DEir-IncRNAs into relative expression, namely IncRNA pairs, to avoid examining specific expression values of every IncRNA. And then, we performed a new method for variable selection and shrinkage in Cox's proportional hazards model-the least absolute shrinkage and selection operator (LASSO), which was deemed more accurate than stepwise selection ${ }^{59}$. Eventually, there were 18 DEir-IncRNA pairs (consisting of 34 DEir-IncRNAs, detail information presented in Table S1) were included in our risk model for prediction of outcome and drug sensitivity in HNSCC patients. Multiple IncRNAs were published the associations among these 34 DEirIncRNAs and human cancers, including HNSCC. The IncRNA LINC00460 plays crucial roles in several epithelial cancers ${ }^{60-62}$, promoting EMT by facilitating peroxiredoxin-1 into the nucleus in HNSCC ${ }^{63}$. The role of LINC02454 was mainly reported in thyroid cancer ${ }^{64-66}$. Abnormal expression of LINC02454 was associated with advanced stage and poor outcome of thyroid cancer ${ }^{66}$. Besides, LINC02454 was deemed as an important immune-related biomarker in auxiliary prognosis prediction for patients with papillary thyroid cancer ${ }^{65}$. High expression of IncRNA SNHG25 promotes cell proliferation, migration and invasion, and decreased apoptosis by up-regulating COMP in epithelial ovarian cancer ${ }^{67}$. In our study, the areas under curve for each receiver operating characteristic (ROC) curve of 18 pairs was drawn to estimate the maximum AUC for survival outcome and to identify optimal cut-off point to differentiate the high- or low risk-group among patients with HNSCC. Our results showed the AUC for 3-year survival of HNSCC is 0.810 , indicating the robust prognosis potential of our risk model. Besides, Kaplan Meier analysis, univariate and multivariate Cox proportional hazard regression analysis authenticated the poor prognosis prediction value of our risk model in HNSCC.

Increasing attention of IncRNAs have been attracted not only for the early screening, diagnosis, prognosis value, but also for the prediction of responses to drug treatment ${ }^{68-70}$. Long non-coding RNA SCAT1 could be served as pretreatment biomarker in prediction pathological response and outcome in esophageal squamous cell carcinoma with neoadjuvant chemoradiotherapy ${ }^{71}$. A stroma-related IncRNA signature (SLS) has been reported to predict adjuvant chemotherapy benefit in patients with colon cancer ${ }^{72}$. LncRNAs are also participated in the acquired resistance to chemotherapy ${ }^{73,74}$, and targeting IncRNA can reverse drug resistance and enhance the sensitivity of cancer cells to chemotherapy ${ }^{75}$. Several studies have indicated that IncRNAs not only involve the typical hallmarks of cancer but also are closely correlated with the regulation of cancer immunity by modulating the immune response ${ }^{76,77}$, these IncRNAs were also associated with the causes of immunotherapy resistance ${ }^{76,78}$. Given the crucial role of IncRNAs in cancer, we also assessed the drug sensitive of our risk, which may provide new insights for 
precise treatment and individualized management of patients with HNSCC. Firstly, we estimated the immune cell infiltration profile, and results showed significant enrichment of B cells naive, plasma cells, $\mathrm{CD}^{+} \mathrm{T}$ cells, $\mathrm{T}$ follicular helper cells (Tfh cells), regulatory $\mathrm{T}$ cells (Treg), mast cells resting in low-risk group, while significant enrichment of CD4+ T cell memory resting, NK cells resting, macrophages $\mathrm{M} 0$ and M2 in high-risk group. T follicular helper (Tfh) cells have been established as a CD4+ subset and expressed a range of cell surface molecules that serve important functions not only in their interactions with B cells but also for their identification like CXC-chemokine receptor 5 (CXCR5) and the coinhibitory receptor programmed cell death 1 (PD1) ${ }^{79-81}$ Treg cells play a major role in maintaining immune homeostasis to keep the balance between the protective and damaging effects of the immune system ${ }^{82,83}$, and recent studies explored that Treg cells is linked to the tumor immune surveillance and carcinogenesis ${ }^{84,85}$. In tumor immunity, Treg cells are involved in tumor development and progression by inhibiting antitumor immunity. There is an interesting phenomenon in our study, namely enrichment of Treg cells in low-risk group of patients with HNSCC based our risk model. Various molecules that are highly expressed in Treg cells, such as immune checkpoint molecules, such as programmed cell death-1 (PD-1) and PD-ligand 1 (PD-L1). Patients with high expression of PD-L1 showed more sensitivity to ICls, such as pembrolizumab and nivolumab; these patients represented favor outcome, which rationalize the above phenomenon. And our prediction drug sensitivity analysis also showed HNSCC patients with lowrisk score represented significantly higher sensitivity to PD-1 and CTLA4.

In summary, we identified an immune-related IncRNAs in HNSCC, and constructed a risk model for prediction prognosis for patients with HNSCC and might help in distinguishing patients who could benefit from anti-tumor immunotherapy.

\section{Methods}

\section{Data download and pretreatment}

The online database TCGA program was aimed to molecularly characterize human cancers. In our study, a series of transcriptome RNA-sequencing data of HNSCC samples were downloaded through GDC portal of TCGA (https://portal.gdc.cancer.gov/), which contained data from 501 HNSCC cases and 44 normal controls. The clinical data was also extracted (including TNM stage, gender, grade, age, survival status and survival data). The long non-coding RNA (IncRNA) data and messenger RNA (mRNA) were extracted from transcriptome RNA-sequencing data, and the annotation file of Ensembl IDs (from http://asia.ensem bl.org/index .html) was applied to convert RNA-seq results into gene symbols.

\section{Immune-related differential expressed IncRNAs acquisition}

Firstly, immune-related genes were obtained from the ImmPort database (http://www.immport.org) and was used to screen immune-related IncRNAs (ir-IncRNAs) by a co-expression strategy. Then, correlation analysis was performed between all IncRNAs of HNSCC samples and known immune-related genes by Pearson's correlation. The cut-off criteria of adjusted $P$-value (adj. P-value) was set as 0.001 and the 
criterion of correlation coefficients was set as more than 0.4. Subsequently, we applied limma package of $\mathrm{R}$ software to identify the differentially expressed ir-IncRNAs. The thresholds were set as log fold change $(F C)>1$ along with false discovery rate $(F D R)<0.05$.

\section{Pairing DEirlncRNAs}

The Cancer Genome Atlas (TCGA) is a comprehensive database that includes multi-layered cancer genome profiles. Large-scale collection of data inevitably generates batch effects introduced by differences in processing at various stages from sample collection to data generation. To eliminate these factors, we cyclically paired these differential expressed ir-IncRNAs (DEir-IncRNAs). We constructed a new 0-or-1 matrix by comparison of all the DEir-IncRNAs expression in each patient of TCGA. We defined the DEir-IncRNA pair as 1 if the expression level of IncRNA A is higher than IncRNA B, otherwise this pair was defined as 0 . This new matrix was used as further analyses.

\section{Survival-related DEir-IncRNAs pair}

Paired DEir-IncRNAs associated with clinical outcomes in HNSCC patients were identified as survivalrelated DEir-IncRNA pairs. Survival-related DEir-IncRNA pairs were selected by univariate Cox regression applying survival package of $\mathrm{R}$ software. These survival-related Deir-IncRNA pairs were specified for subsequent research.

\section{Establishment of a risk model}

The least absolute shrinkage and selection operator (LASSO) is a regression-based methodology permitting for a large number of covariates in the model, and importantly has the unique feature penalizing the absolute value of a regression coefficient; thus, regulating the impact a coefficient may have on the overall regression. The greater the penalization, the greater the shrinkage of coefficients, with some reaching 0 , thus automatically removing unnecessary/uninfluential covariates. The survival-related DEir-IncRNA pairs were selected for Lasso regression and Cox proportional hazard regression analysis, as well as for construction of the model. Risk score is a simplified version of a prognostic model, in which scores are assigned to each risk factor (on the basis of rounded regression coefficients). The risk score of each sample was calculated by the following formula:

Risk score $=$ coefficient $_{1} *$ DEir-IncRNA pair $_{1}+$ coefficient $_{2}{ }^{*}$ expression of $($ DEir-IncRNA

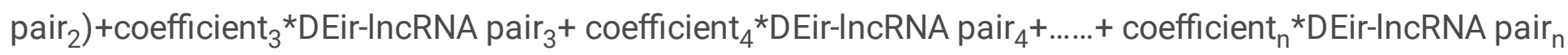

The value of coefficient was generated from Cox proportional hazard regression analysis. The 1-, 2-, 3-, 4-, 5- and 6-year receiver operating characteristic (ROC) curves of the model were cyclically plotted till the maximum areas under curve for each ROC (AUC) were obtained. Then, the highest point of risk score of maximum AUC were set to the cut-off criteria to classify HNSCC patients into high or low risk of group.

\section{Validation of the constructed risk model}


To validate the constructed risk model, we performed Kaplan-Meier analysis to show the survival difference of high and low risk group. Subsequently, we also re-assessed the relationship between the model and clinicopathological characteristics. Then, to confirm whether the model can be used as an independent clinical prognostic biomarker, we performed the univariate and multivariate Cox regression analyses, and the results were represented by forest map.

\section{Immune cell abundance analysis}

We collected the currently acknowledged methods to calculate the immune infiltration statues from CIBERSORT ${ }^{86}$. The results were shown in a lollipop diagram, which was finished by violet package of $\mathrm{R}$ software.

\section{Prediction of clinical application of the risk model}

Recent studies have predicted the response to immune checkpoint inhibitors (ICls) of cancers based on their genomic features, and the immunophenoscore (IPS) was correlated with responses to ICls immunotherapy ${ }^{87,88}$. The score of IPS-CTLA-4 and IPS-PD1 stands for the potential for response rates of ICls in HNSCC. Besides, we calculated a half inhibitory centration $\left(\mathrm{IC}_{50}\right)$ of common chemotherapeutic drugs, such as cisplatin, docetaxel, gemcitabine and paclitaxel. The difference in the IC50 between the high and low risk groups were assessed and the results were shown as box drawings by pRRophetic package of $\mathrm{R}^{89}$. Then, we also analyzed the relationship between the model and the expression of $\mathrm{ICI}$ related genes.

\section{Declarations}

\section{Data Availability Statement}

The datasets analyzed during the current study are available in the TCGA database (https://portal.gdc.cancer.gov/).

\section{Author Contributions}

All authors contributed to the planning and design of the study. CZ and JL were involved in review of the raw data and directly involved in the analysis. ZS, YS and YMS provided analytical feedback based on aggregated results. CZ, JL and GZ drafted the manuscript, with input from all authors. JW, HD and YMS were responsible for the chart making. All authors provided substantive review and commentary on multiple drafts and approved the final version. $\mathrm{JL}$ and $\mathrm{GZ}$ had full access to all of the data in the study and takes responsibility for the integrity of the data and the accuracy of the data analysis.

\section{Conflict of interest}

The authors declare that there are no conflicts of interest. 


\section{Funding}

The National Natural Science Foundation of China (No.81670920), Zhejiang Provincial Natural Science Foundation of China (No. LY19H160014 and No. LQ21H130001), Ningbo medical and health brand discipline (No. PPXK2018-02), Zhejiang Province Medical and Health Research Project (No. 2019ZD018, No. 2020RC107, and No. 2021KY307), Ningbo Natural Science Foundation (No. 202003N4239), Ningbo Huimin Technology Research and Development Project Fund (No. 2015C50026), and Ningbo "Technology Innovation 2025" Major Special Project (No. $2020 Z 097$ and No. 2018B10013) supported this work.

\section{References}

1 Chow, L. Q. M. Head and Neck Cancer. N Engl J Med 382, 60-72, doi:10.1056/NEJMra1715715 (2020).

2 Gillison, M. L., Chaturvedi, A. K., Anderson, W. F. \& Fakhry, C. Epidemiology of Human Papillomavirus-Positive Head and Neck Squamous Cell Carcinoma. J Clin Onco/33, 3235-3242, doi:10.1200/JC0.2015.61.6995 (2015).

3 Hashibe, M. et al. Alcohol drinking in never users of tobacco, cigarette smoking in never drinkers, and the risk of head and neck cancer: pooled analysis in the International Head and Neck Cancer Epidemiology Consortium. J Natl Cancer Inst 99, 777-789, doi:10.1093/jnci/djk179 (2007).

$4 \quad$ Wyss, A. et al. Cigarette, cigar, and pipe smoking and the risk of head and neck cancers: pooled analysis in the International Head and Neck Cancer Epidemiology Consortium. Am J Epidemio/ 178, 679690, doi:10.1093/aje/kwt029 (2013).

5 McDermott, J. D. \& Bowles, D. W. Epidemiology of Head and Neck Squamous Cell Carcinomas: Impact on Staging and Prevention Strategies. Curr Treat Options Oncol 20, 43, doi:10.1007/s11864-0190650-5 (2019).

6 Hanahan, D. \& Weinberg, R. A. Hallmarks of cancer: the next generation. Cel/ 144, 646-674, doi:10.1016/j.cell.2011.02.013 (2011).

7 Cho, Y., Milane, L. \& Amiji, M. M. Genetic and epigenetic strategies for advancing ovarian cancer immunotherapy. Expert Opin Biol Ther 19, 547-560, doi:10.1080/14712598.2019.1602605 (2019).

8 Costa-Pinheiro, P., Montezuma, D., Henrique, R. \& Jeronimo, C. Diagnostic and prognostic epigenetic biomarkers in cancer. Epigenomics 7, 1003-1015, doi:10.2217/epi.15.56 (2015).

9 Qian, Y. et al. Molecular alterations and targeted therapy in pancreatic ductal adenocarcinoma. $J$ Hematol Oncol 13, 130, doi:10.1186/s13045-020-00958-3 (2020).

10 Boon, T., Cerottini, J. C., Van den Eynde, B., van der Bruggen, P. \& Van Pel, A. Tumor antigens recognized by T lymphocytes. Annu Rev Immuno/ 12, 337-365, doi:10.1146/annurev.iy.12.040194.002005 
(1994).

11 Fleming, C., Morrissey, S., Cai, Y. \& Yan, J. gammadelta T Cells: Unexpected Regulators of Cancer Development and Progression. Trends Cancer 3, 561-570, doi:10.1016/j.trecan.2017.06.003 (2017).

12 Marshall, E. A. et al. Emerging roles of T helper 17 and regulatory T cells in lung cancer progression and metastasis. Mol Cancer 15, 67, doi:10.1186/s12943-016-0551-1 (2016).

13 O'Donnell, J. S., Teng, M. W. L. \& Smyth, M. J. Cancer immunoediting and resistance to T cellbased immunotherapy. Nat Rev Clin Oncol 16, 151-167, doi:10.1038/s41571-018-0142-8 (2019).

14 Banchereau, J. \& Steinman, R. M. Dendritic cells and the control of immunity. Nature 392, 245-252, doi:10.1038/32588 (1998).

15 Gardner, A. \& Ruffell, B. Dendritic Cells and Cancer Immunity. Trends Immuno/ 37, 855-865, doi:10.1016/j.it.2016.09.006 (2016).

16 Hilligan, K. L. \& Ronchese, F. Antigen presentation by dendritic cells and their instruction of CD4+ T helper cell responses. Cell Mol Immuno/ 17, 587-599, doi:10.1038/s41423-020-0465-0 (2020).

17 Mullard, A. New checkpoint inhibitors ride the immunotherapy tsunami. Nat Rev Drug Discov 12, 489-492, doi:10.1038/nrd4066 (2013).

18 McCune, J. S. Rapid Advances in Immunotherapy to Treat Cancer. Clin Pharmacol Ther 103, 540544, doi:10.1002/cpt.985 (2018).

19 Predina, J. et al. Changes in the local tumor microenvironment in recurrent cancers may explain the failure of vaccines after surgery. Proc Natl Acad Sci U S A 110, E415-424, doi:10.1073/pnas.1211850110 (2013).

20 Wang, L. et al. Immune evasion of mantle cell lymphoma: expression of B7-H1 leads to inhibited Tcell response to and killing of tumor cells. Haematologica 98, 1458-1466, doi:10.3324/haematol.2012.071340 (2013).

21 Chen, D. S., Irving, B. A. \& Hodi, F. S. Molecular pathways: next-generation immunotherapy-inhibiting programmed death-ligand 1 and programmed death-1. Clin Cancer Res 18, 6580-6587, doi:10.1158/1078-0432.CCR-12-1362 (2012).

22 Motz, G. T. \& Coukos, G. Deciphering and reversing tumor immune suppression. Immunity 39, 6173, doi:10.1016/j.immuni.2013.07.005 (2013).

23 Khalil, A. M. et al. Many human large intergenic noncoding RNAs associate with chromatinmodifying complexes and affect gene expression. Proc Natl Acad Sci U S A 106, 11667-11672, doi:10.1073/pnas.0904715106 (2009). 
24 Mathy, N. W. \& Chen, X. M. Long non-coding RNAs (IncRNAs) and their transcriptional control of inflammatory responses. J Biol Chem 292, 12375-12382, doi:10.1074/jbc.R116.760884 (2017).

25 Bartonicek, N., Maag, J. L. \& Dinger, M. E. Long noncoding RNAs in cancer: mechanisms of action and technological advancements. Mol Cancer 15, 43, doi:10.1186/s12943-016-0530-6 (2016).

26 Kornfeld, J. W. \& Bruning, J. C. Regulation of metabolism by long, non-coding RNAs. Front Genet 5, 57, doi:10.3389/fgene.2014.00057 (2014).

27 Vitiello, M., Tuccoli, A. \& Poliseno, L. Long non-coding RNAs in cancer: implications for personalized therapy. Cell Oncol (Dordr) 38, 17-28, doi:10.1007/s13402-014-0180-x (2015).

28 Fu, X., Ravindranath, L., Tran, N., Petrovics, G. \& Srivastava, S. Regulation of apoptosis by a prostate-specific and prostate cancer-associated noncoding gene, PCGEM1. DNA Cell Bio/ 25, 135-141, doi:10.1089/dna.2006.25.135 (2006).

29 Walsh, A. L., Tuzova, A. V., Bolton, E. M., Lynch, T. H. \& Perry, A. S. Long noncoding RNAs and prostate carcinogenesis: the missing 'linc'? Trends Mol Med 20, 428-436, doi:10.1016/j.molmed.2014.03.005 (2014).

30 Warrick, J. I. et al. Evaluation of tissue PCA3 expression in prostate cancer by RNA in situ hybridization-a correlative study with urine PCA3 and TMPRSS2-ERG. Mod Pathol 27, 609-620, doi:10.1038/modpathol.2013.169 (2014).

31 Bhan, A. et al. Antisense transcript long noncoding RNA (IncRNA) HOTAIR is transcriptionally induced by estradiol. J Mol Biol 425, 3707-3722, doi:10.1016/j.jmb.2013.01.022 (2013).

32 Geng, Y. J., Xie, S. L., Li, Q., Ma, J. \& Wang, G. Y. Large intervening non-coding RNA HOTAIR is associated with hepatocellular carcinoma progression. J Int Med Res 39, 2119-2128, doi:10.1177/147323001103900608 (2011).

$33 \mathrm{Kim}, \mathrm{K}$. et al. HOTAIR is a negative prognostic factor and exhibits pro-oncogenic activity in pancreatic cancer. Oncogene 32, 1616-1625, doi:10.1038/onc.2012.193 (2013).

$34 \mathrm{Li}$, D. et al. Long intergenic noncoding RNA HOTAIR is overexpressed and regulates PTEN methylation in laryngeal squamous cell carcinoma. Am J Pathol 182, 64-70, doi:10.1016/j.ajpath.2012.08.042 (2013).

35 Nakagawa, T. et al. Large noncoding RNA HOTAIR enhances aggressive biological behavior and is associated with short disease-free survival in human non-small cell lung cancer. Biochem Biophys Res Commun 436, 319-324, doi:10.1016/j.bbrc.2013.05.101 (2013).

36 Fatica, A. \& Bozzoni, I. Long non-coding RNAs: new players in cell differentiation and development. Nat Rev Genet 15, 7-21, doi:10.1038/nrg3606 (2014). 
37 Yu, W. D., Wang, H., He, Q. F., Xu, Y. \& Wang, X. C. Long noncoding RNAs in cancer-immunity cycle. J Cell Physio/ 233, 6518-6523, doi:10.1002/jcp.26568 (2018).

38 Pandya, P. H., Murray, M. E., Pollok, K. E. \& Renbarger, J. L. The Immune System in Cancer Pathogenesis: Potential Therapeutic Approaches. J Immunol Res 2016, 4273943, doi:10.1155/2016/4273943 (2016).

39 Zhou, D. et al. Macrophage polarization and function with emphasis on the evolving roles of coordinated regulation of cellular signaling pathways. Cell Signa/ 26, 192-197, doi:10.1016/j.cellsig.2013.11.004 (2014).

40 Huang, Z. et al. Identification of Differentially Expressed Long Non-coding RNAs in Polarized Macrophages. Sci Rep 6, 19705, doi:10.1038/srep19705 (2016).

41 Ye, Y. et al. Long non-coding RNA cox-2 prevents immune evasion and metastasis of hepatocellular carcinoma by altering M1/M2 macrophage polarization. J Cell Biochem 119, 2951-2963, doi:10.1002/jcb.26509 (2018).

42 Fang, P. et al. LncRNA GAS5 enhanced the killing effect of NK cell on liver cancer through regulating miR-544/RUNX3. Innate Immun 25, 99-109, doi:10.1177/1753425919827632 (2019).

43 Ou, Z. L. et al. Hypoxia-induced shedding of MICA and HIF1A-mediated immune escape of pancreatic cancer cells from NK cells: role of circ_0000977/miR-153 axis. RNA Bio/ 16, 1592-1603, doi:10.1080/15476286.2019.1649585 (2019).

44 Wei, M. F., Gu, Z. S., Zheng, L. L., Zhao, M. X. \& Wang, X. J. Long non-coding RNA GAS5 promotes natural killer cell cytotoxicity against gastric cancer by regulating miR-18a. Neoplasma 67, 1085-1093, doi:10.4149/neo_2020_191014N1034 (2020).

45 Zhang, Y., Li, X., Zhang, J. \& Liang, H. Natural killer T cell cytotoxic activity in cervical cancer is facilitated by the LINC00240/microRNA-124-3p/STAT3/MICA axis. Cancer Lett 474, 63-73, doi:10.1016/j.canlet.2019.12.038 (2020).

46 Newman, A. M. et al. Determining cell type abundance and expression from bulk tissues with digital cytometry. Nat Biotechno/ 37, 773-782, doi:10.1038/s41587-019-0114-2 (2019).

47 Bauml, J. et al. Pembrolizumab for Platinum- and Cetuximab-Refractory Head and Neck Cancer: Results From a Single-Arm, Phase II Study. J Clin Onco/35, 1542-1549, doi:10.1200/JC0.2016.70.1524 (2017).

48 Cohen, E. E. W. et al. Pembrolizumab versus methotrexate, docetaxel, or cetuximab for recurrent or metastatic head-and-neck squamous cell carcinoma (KEYNOTE-040): a randomised, open-label, phase 3 study. Lancet 393, 156-167, doi:10.1016/S0140-6736(18)31999-8 (2019). 
49 Mehra, R. et al. Efficacy and safety of pembrolizumab in recurrent/metastatic head and neck squamous cell carcinoma: pooled analyses after long-term follow-up in KEYNOTE-012. Br J Cancer 119, 153-159, doi:10.1038/s41416-018-0131-9 (2018).

50 Pai, S. I. et al. Comparative analysis of the phase III clinical trials of anti-PD1 monotherapy in head and neck squamous cell carcinoma patients (CheckMate 141 and KEYNOTE 040). J Immunother Cancer 7, 96, doi:10.1186/s40425-019-0578-0 (2019).

51 Seiwert, T. Y. et al. Safety and clinical activity of pembrolizumab for treatment of recurrent or metastatic squamous cell carcinoma of the head and neck (KEYNOTE-012): an open-label, multicentre, phase 1b trial. Lancet Oncol 17, 956-965, doi:10.1016/S1470-2045(16)30066-3 (2016).

52 Rizvi, H. et al. Molecular Determinants of Response to Anti-Programmed Cell Death (PD)-1 and Anti-Programmed Death-Ligand 1 (PD-L1) Blockade in Patients With Non-Small-Cell Lung Cancer Profiled With Targeted Next-Generation Sequencing. J Clin Onco/ 36, 633-641, doi:10.1200/JC0.2017.75.3384 (2018).

53 Liu, A. N., Qu, H. J., Yu, C. Y. \& Sun, P. Knockdown of LINC01614 inhibits lung adenocarcinoma cell progression by up-regulating miR-217 and down-regulating FOXP1. J Cell Mol Med 22, 4034-4044, doi:10.1111/jcmm.13483 (2018).

54 Vishnubalaji, R., Shaath, H., Elkord, E. \& Alajez, N. M. Long non-coding RNA (IncRNA) transcriptional landscape in breast cancer identifies LINC01614 as non-favorable prognostic biomarker regulated by TGFbeta and focal adhesion kinase (FAK) signaling. Cell Death Discov 5, 109, doi:10.1038/s41420-019-0190-6 (2019).

55 Eble, J. A. \& Niland, S. The extracellular matrix in tumor progression and metastasis. Clin Exp Metastasis 36, 171-198, doi:10.1007/s10585-019-09966-1 (2019).

56 Pickup, M. W., Mouw, J. K. \& Weaver, V. M. The extracellular matrix modulates the hallmarks of cancer. EMBO Rep 15, 1243-1253, doi:10.15252/embr.201439246 (2014).

57 Walker, C., Mojares, E. \& Del Rio Hernandez, A. Role of Extracellular Matrix in Development and Cancer Progression. Int J Mol Sci 19, doi:10.3390/ijms19103028 (2018).

58 Qing, L. et al. Extracellular Matrix-Related Six-IncRNA Signature as a Novel Prognostic Biomarker for Bladder Cancer. Onco Targets Ther 13, 12521-12538, doi:10.2147/OTT.S284167 (2020).

59 Tibshirani, R. The lasso method for variable selection in the Cox model. Stat Med 16, 385-395, doi:10.1002/(sici)1097-0258(19970228)16:4<385::aid-sim380>3.0.co;2-3 (1997).

60 Cui, Y. et al. LncRNA linc00460 sponges miR-1224-5p to promote esophageal cancer metastatic potential and epithelial-mesenchymal transition. Pathol Res Pract 216, 153026, doi:10.1016/j.prp.2020.153026 (2020). 
61 Dong, Y. \& Quan, H. Y. Downregulated LINC00460 inhibits cell proliferation and promotes cell apoptosis in prostate cancer. Eur Rev Med Pharmacol Sci 23, 6070-6078, doi:10.26355/eurrev_201907_18420 (2019).

62 Zhao, H., Wang, Y. \& Ren, X. Nicotine promotes the development of non-small cell lung cancer through activating LINC00460 and PI3K/Akt signaling. Biosci Rep 39, doi:10.1042/BSR20182443 (2019).

63 Jiang, Y. et al. LncRNA LINC00460 promotes EMT in head and neck squamous cell carcinoma by facilitating peroxiredoxin-1 into the nucleus. J Exp Clin Cancer Res 38, 365, doi:10.1186/s13046-0191364-z (2019).

64 Cai, W. Y. et al. Role of differentially expressed genes and long non-coding RNAs in papillary thyroid carcinoma diagnosis, progression, and prognosis. J Cell Biochem 119, 8249-8259, doi:10.1002/jcb.26836 (2018).

$65 \mathrm{Li}, \mathrm{Z}$. et al. Identification of immune-related IncRNAs to improve the prognosis prediction for patients with papillary thyroid cancer. Biosci Rep 41, doi:10.1042/BSR20204086 (2021).

66 Tan, J. et al. Overexpression of novel long intergenic noncoding RNA LINC02454 is associated with a poor prognosis in papillary thyroid cancer. Oncol Rep 44, 1489-1501, doi:10.3892/or.2020.7712 (2020).

67 Liu, Y. et al. Long non-coding RNA SNHG25 promotes epithelial ovarian cancer progression by upregulating COMP. J Cancer 12, 1660-1668, doi:10.7150/jca.47344 (2021).

68 Necula, L. et al. Recent advances in gastric cancer early diagnosis. World J Gastroentero/ 25, 20292044, doi:10.3748/wjg.v25.i17.2029 (2019).

69 Zhang, E. et al. A novel long noncoding RNA HOXC-AS3 mediates tumorigenesis of gastric cancer by binding to YBX1. Genome Bio/ 19, 154, doi:10.1186/s13059-018-1523-0 (2018).

70 Zhuo, W. et al. Long Noncoding RNA GMAN, Up-regulated in Gastric Cancer Tissues, Is Associated With Metastasis in Patients and Promotes Translation of Ephrin A1 by Competitively Binding GMAN-AS. Gastroenterology 156, 676-691 e611, doi:10.1053/j.gastro.2018.10.054 (2019).

71 Zhang, C. et al. A three-IncRNA signature of pretreatment biopsies predicts pathological response and outcome in esophageal squamous cell carcinoma with neoadjuvant chemoradiotherapy. Clin Transl Med 10, e156, doi:10.1002/ctm2.156 (2020).

72 Zhou, R. et al. A stroma-related IncRNA panel for predicting recurrence and adjuvant chemotherapy benefit in patients with early-stage colon cancer. J Cell Mol Med 24, 3229-3241, doi:10.1111/jcmm.14999 (2020). 
73 Huan, L. et al. Hypoxia induced LUCAT1/PTBP1 axis modulates cancer cell viability and chemotherapy response. Mol Cancer 19, 11, doi:10.1186/s12943-019-1122-z (2020).

74 Yang, Q. et al. IncRNA SLC7A11-AS1 Promotes Chemoresistance by Blocking SCF(beta-TRCP)Mediated Degradation of NRF2 in Pancreatic Cancer. Mol Ther Nucleic Acids 19, 974-985, doi:10.1016/j.omtn.2019.11.035 (2020).

$75 \mathrm{Gu}, \mathrm{N}$. et al. Silencing IncRNA FOXD2-AS1 inhibits proliferation, migration, invasion and drug resistance of drug-resistant glioma cells and promotes their apoptosis via microRNA-98-5p/CPEB4 axis. Aging (Albany NY) 11, 10266-10283, doi:10.18632/aging.102455 (2019).

76 Fares, C. M., Van Allen, E. M., Drake, C. G., Allison, J. P. \& Hu-Lieskovan, S. Mechanisms of Resistance to Immune Checkpoint Blockade: Why Does Checkpoint Inhibitor Immunotherapy Not Work for All Patients? Am Soc Clin Oncol Educ Book 39, 147-164, doi:10.1200/EDBK_240837 (2019).

77 Wei, B., Kong, W., Mou, X. \& Wang, S. Comprehensive analysis of tumor immune infiltration associated with endogenous competitive RNA networks in lung adenocarcinoma. Pathol Res Pract 215, 159-170, doi:10.1016/j.prp.2018.10.032 (2019).

78 Heward, J. A. \& Lindsay, M. A. Long non-coding RNAs in the regulation of the immune response. Trends Immuno/ 35, 408-419, doi:10.1016/j.it.2014.07.005 (2014).

79 Crotty, S. T Follicular Helper Cell Biology: A Decade of Discovery and Diseases. Immunity 50, 11321148, doi:10.1016/j.immuni.2019.04.011 (2019).

80 Hetta, H. F. et al. T follicular helper and T follicular regulatory cells in colorectal cancer: A complex interplay. J Immunol Methods 480, 112753, doi:10.1016/j.jim.2020.112753 (2020).

81 Vinuesa, C. G., Linterman, M. A., Yu, D. \& MacLennan, I. C. Follicular Helper T Cells. Annu Rev Immuno/34, 335-368, doi:10.1146/annurev-immunol-041015-055605 (2016).

82 Hetta, H. F. et al. Association of colonic regulatory T cells with hepatitis C virus pathogenesis and liver pathology. J Gastroenterol Hepato/ 30, 1543-1551, doi:10.1111/jgh.12936 (2015).

83 Mehta, M. et al. Association between IL28B rs12979860 single nucleotide polymorphism and the frequency of colonic Treg in chronically HCV-infected patients. Arch Viro/ 161, 3161-3169, doi:10.1007/s00705-016-3015-4 (2016).

84 Zahran, A. M. et al. Up-regulation of regulatory T cells, CD200 and TIM3 expression in cytogenetically normal acute myeloid leukemia. Cancer Biomark 22, 587-595, doi:10.3233/CBM-181368 (2018).

85 Zahran, A. M. et al. Increased frequency and FOXP3 expression of human CD8(+)CD25(High+) T lymphocytes and its relation to CD4 regulatory $T$ cells in patients with hepatocellular carcinoma. Hum 
Immuno/ 80, 510-516, doi:10.1016/j.humimm.2019.03.014 (2019).

86 Kawada, J. I. et al. Immune cell infiltration landscapes in pediatric acute myocarditis analyzed by CIBERSORT. J Cardio/ 77, 174-178, doi:10.1016/j.jjcc.2020.08.004 (2021).

87 Hugo, W. et al. Genomic and Transcriptomic Features of Response to Anti-PD-1 Therapy in Metastatic Melanoma. Cell 165, 35-44, doi:10.1016/j.cell.2016.02.065 (2016).

88 Van Allen, E. M. et al. Genomic correlates of response to CTLA-4 blockade in metastatic melanoma. Science 350, 207-211, doi:10.1126/science.aad0095 (2015).

89 Geeleher, P., Cox, N. \& Huang, R. S. pRRophetic: an R package for prediction of clinical chemotherapeutic response from tumor gene expression levels. PLoS One 9, e107468, doi:10.1371/journal.pone.0107468 (2014).

\section{Figures}
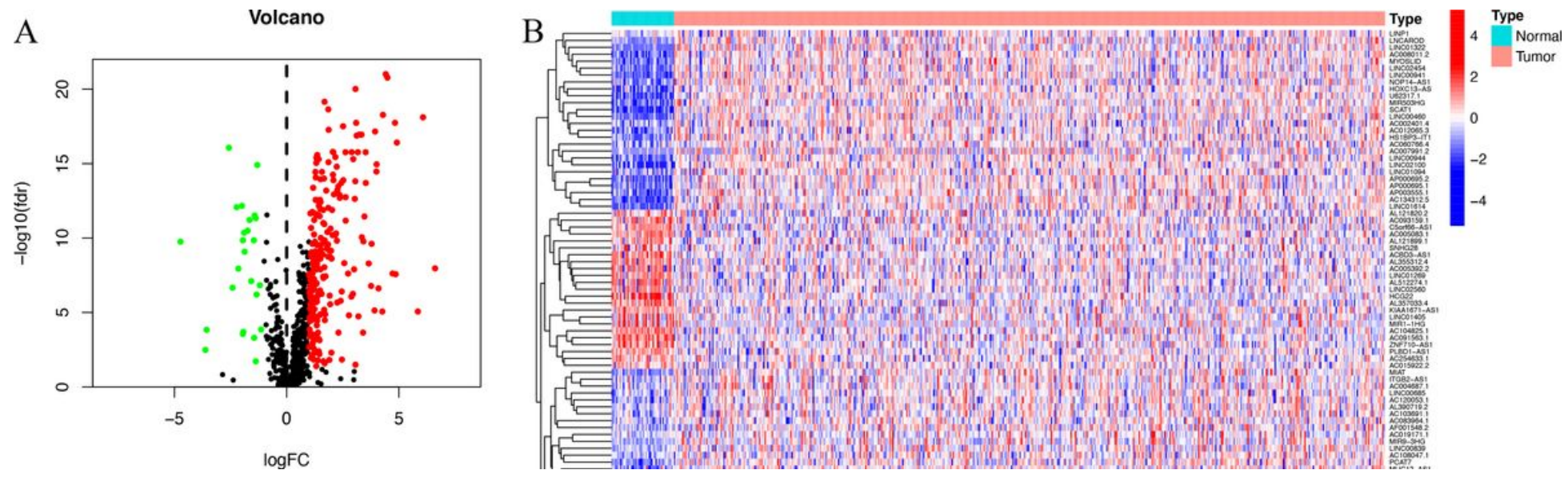

Figure 1

Volcano (1A) and heatmep (1B) plots of DEir-IncRNAs of HNSCC. 


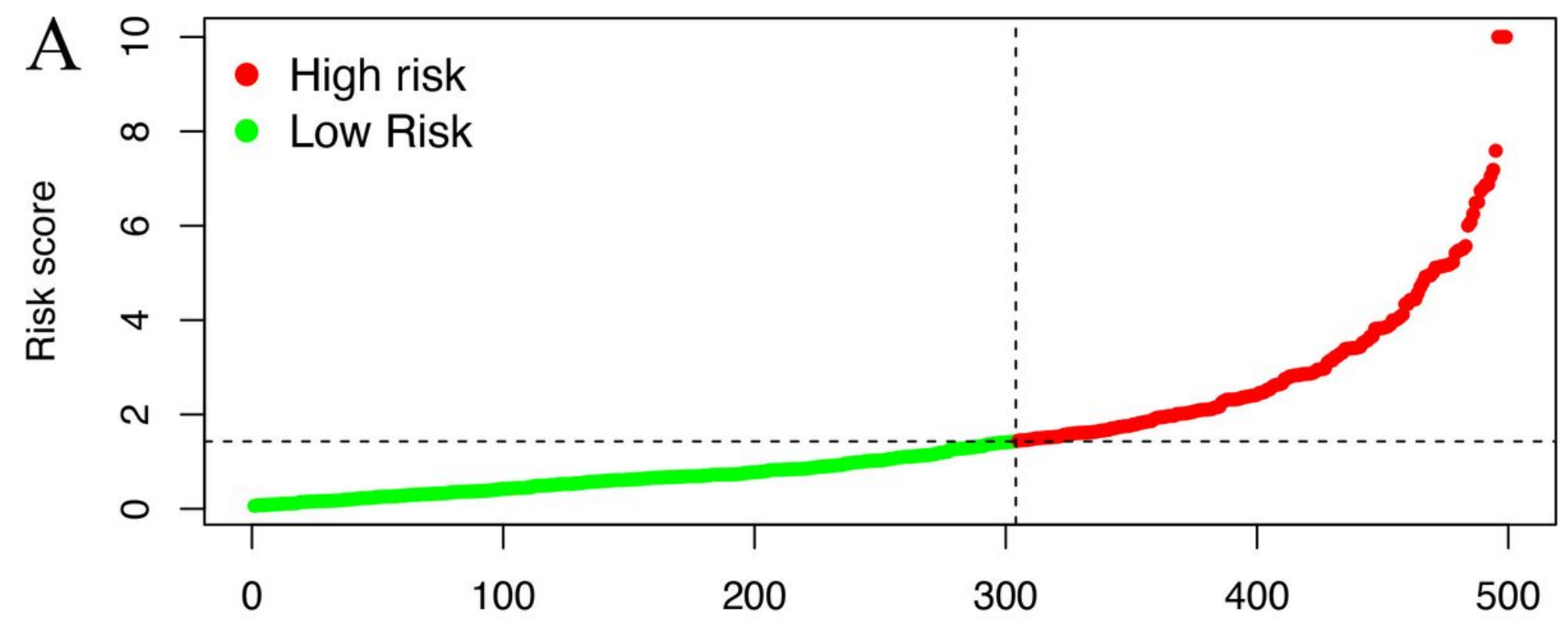

Patients (increasing risk socre)

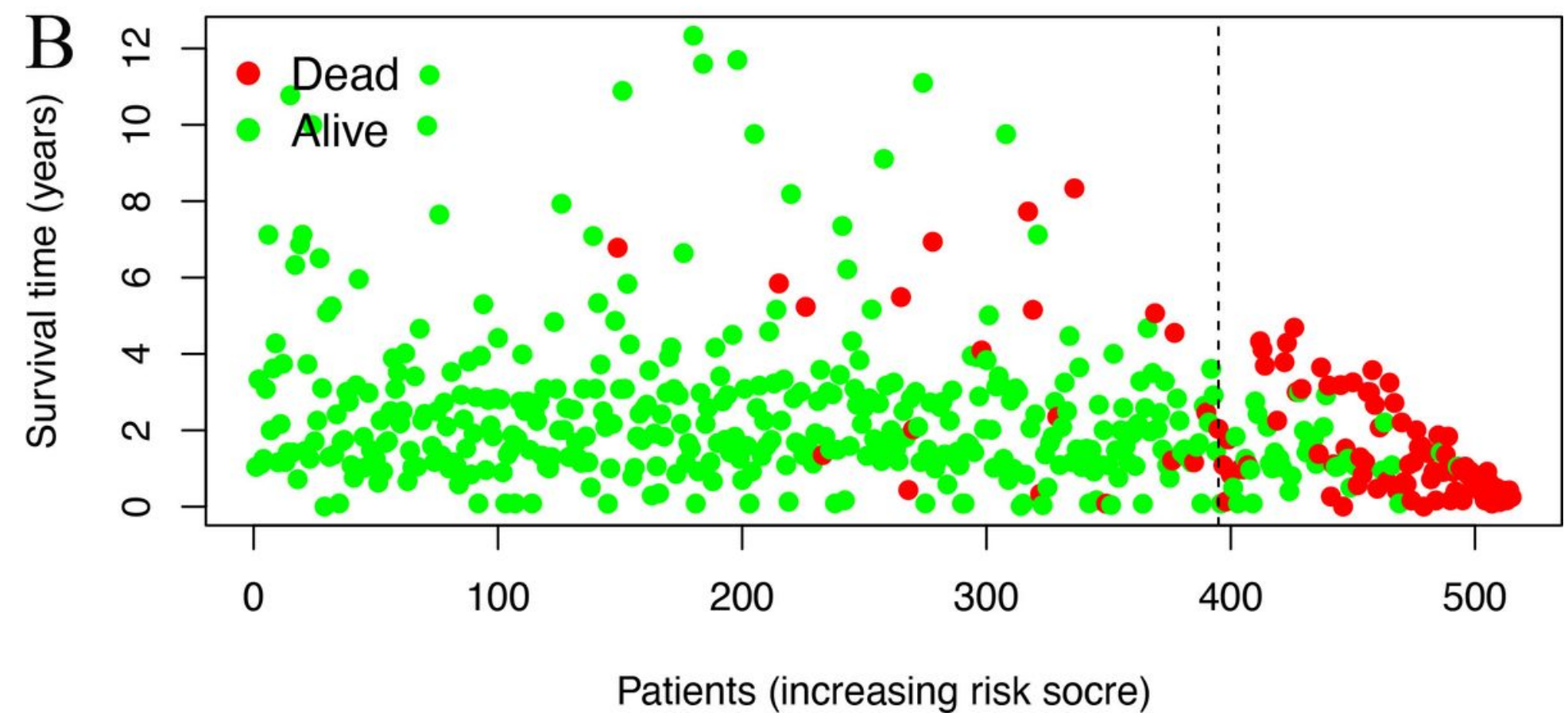

Figure 2

Risk scores of each HNSCC patient based on risk model. 


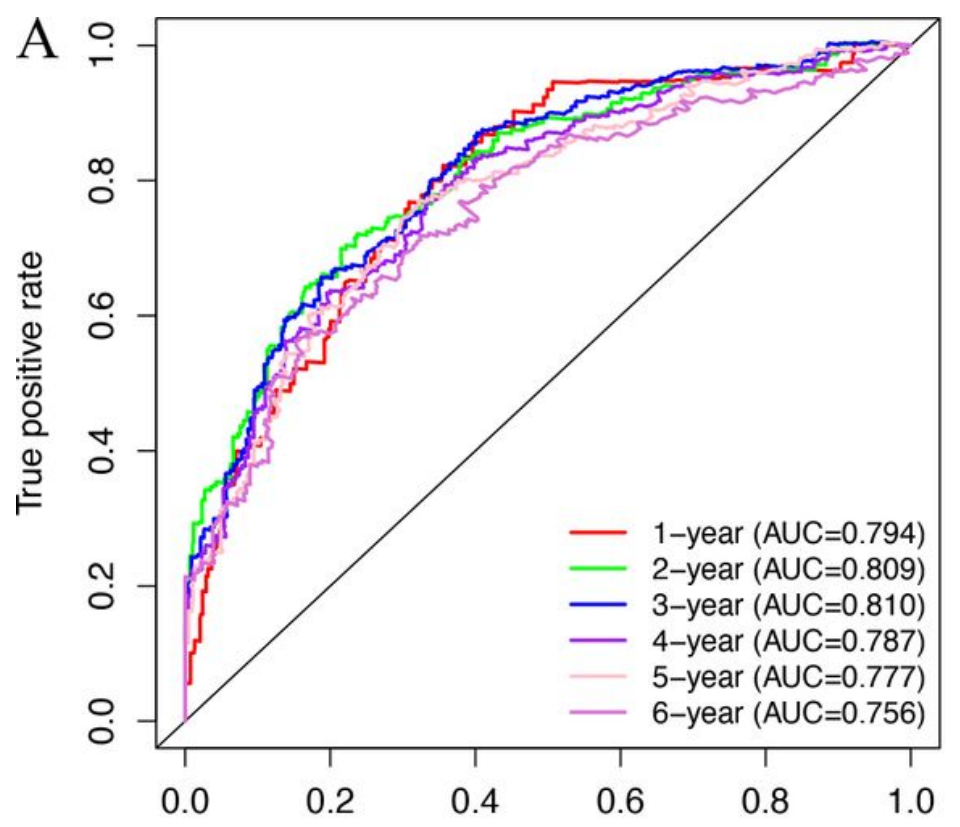

False positive rate

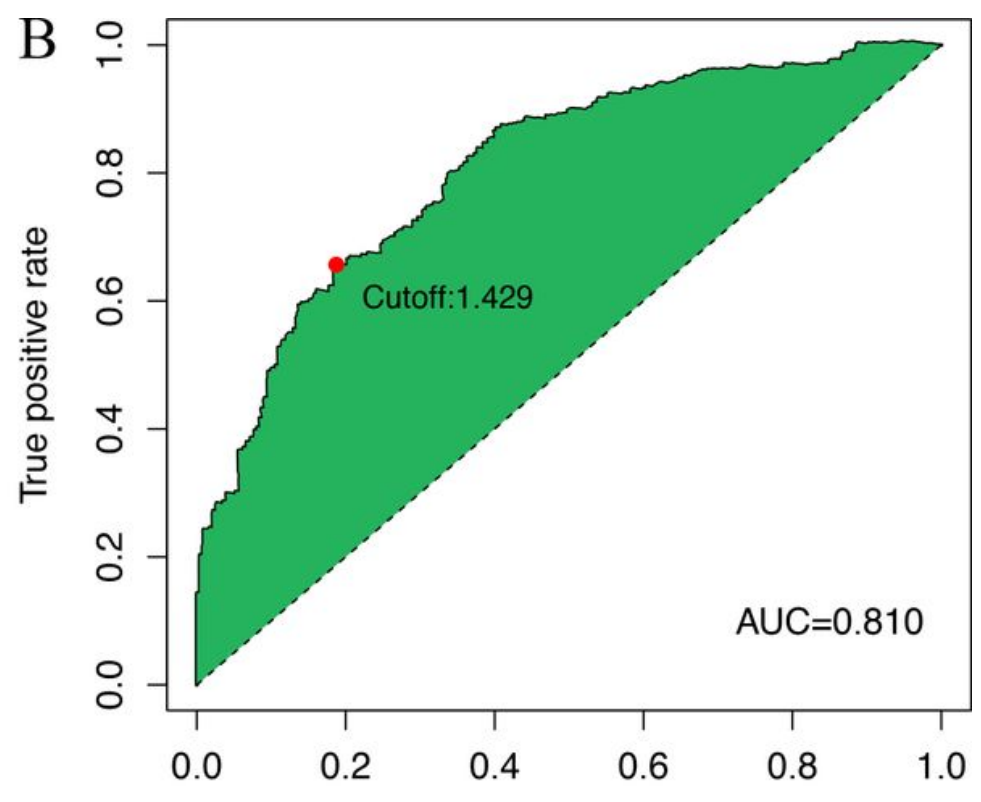

False positive rate

\section{Figure 3}

Time-dependent receiver operating characteristic (ROC) analysis at1-, 2-, 3-, 4-, 5- and 6 years to identify the maximum of the AUC (3A) and to identify the optimal cut-off point to group HNSCC case (3B).

Risk + All + High risk + Low risk
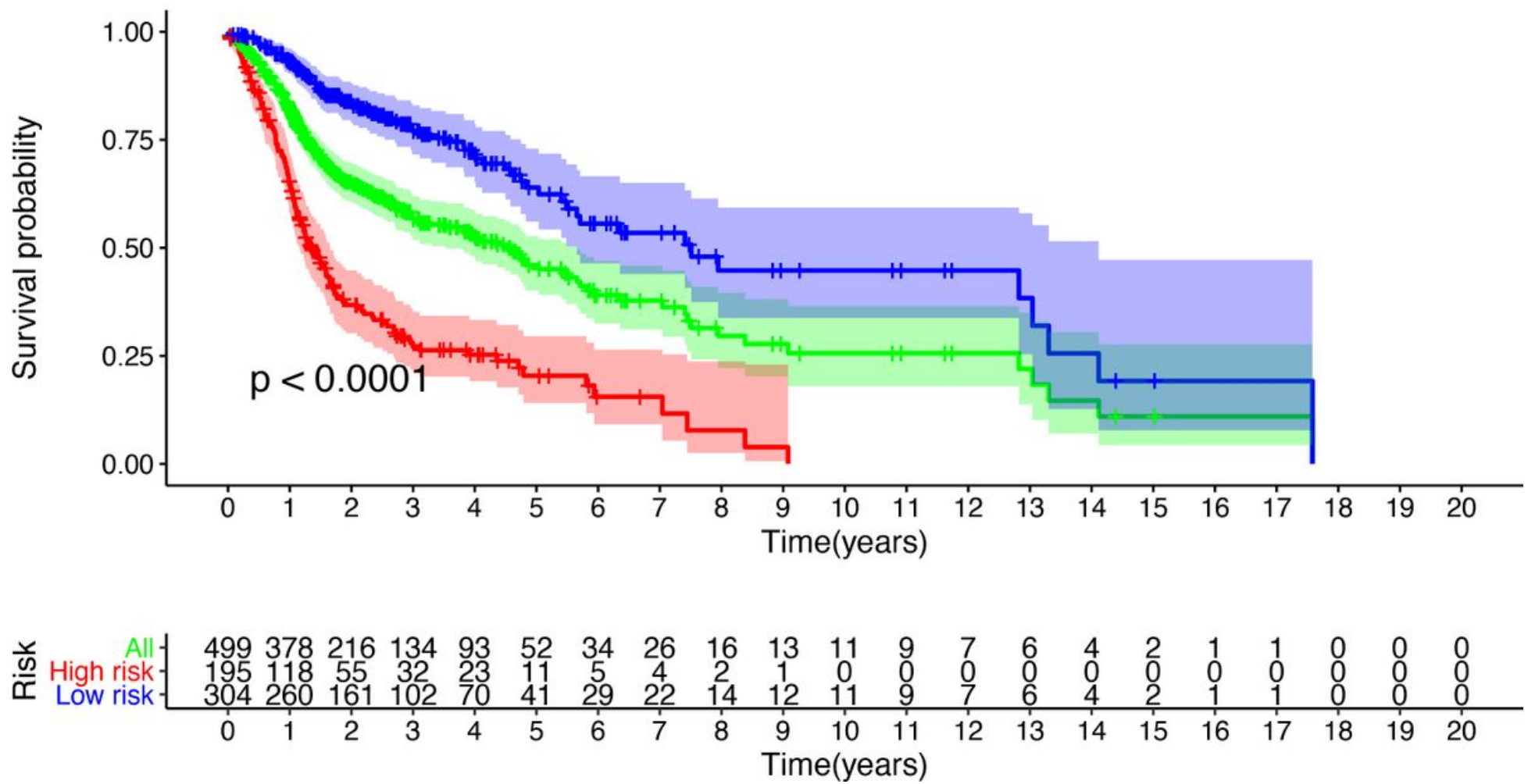

\section{Figure 4}

The Kaplan-Meier plot of HNSCC grouped by risk model. 
A

$\begin{array}{lll}\text { Age } & <0.001 & 1.024(1.010-1.038) \\ \text { Gender } & 0.114 & 0.776(0.566-1.063) \\ \text { Grade } & 0.225 & 1.152(0.917-1.449) \\ \text { Stage } & <0.001 & 1.448(1.203-1.743) \\ & & \\ \text { riskScore }<0.001 & 1.437(1.362-1.515)\end{array}$

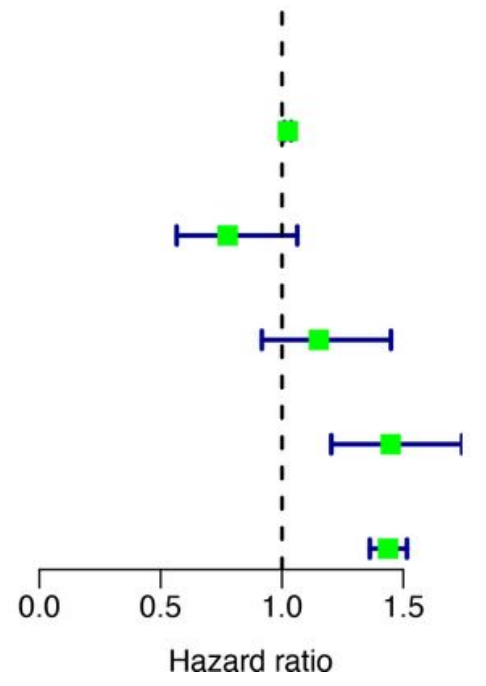

$\mathrm{B}$

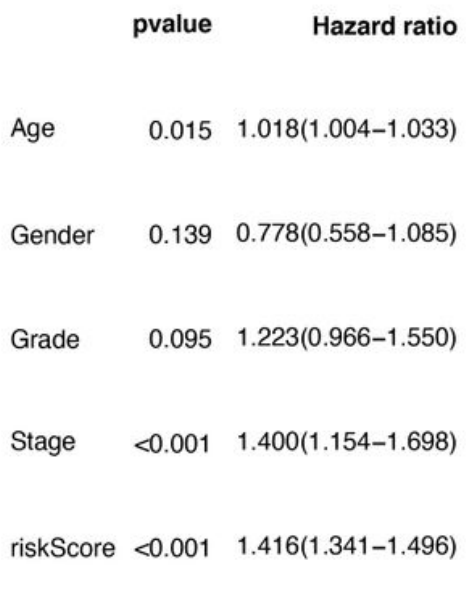

multivariate

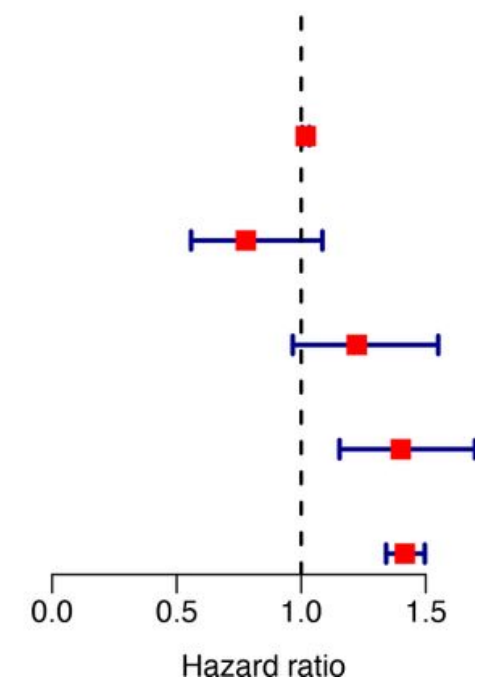

Figure 5

Univariate and multivariate Cox proportional hazard regression of risk model and other clinical features.

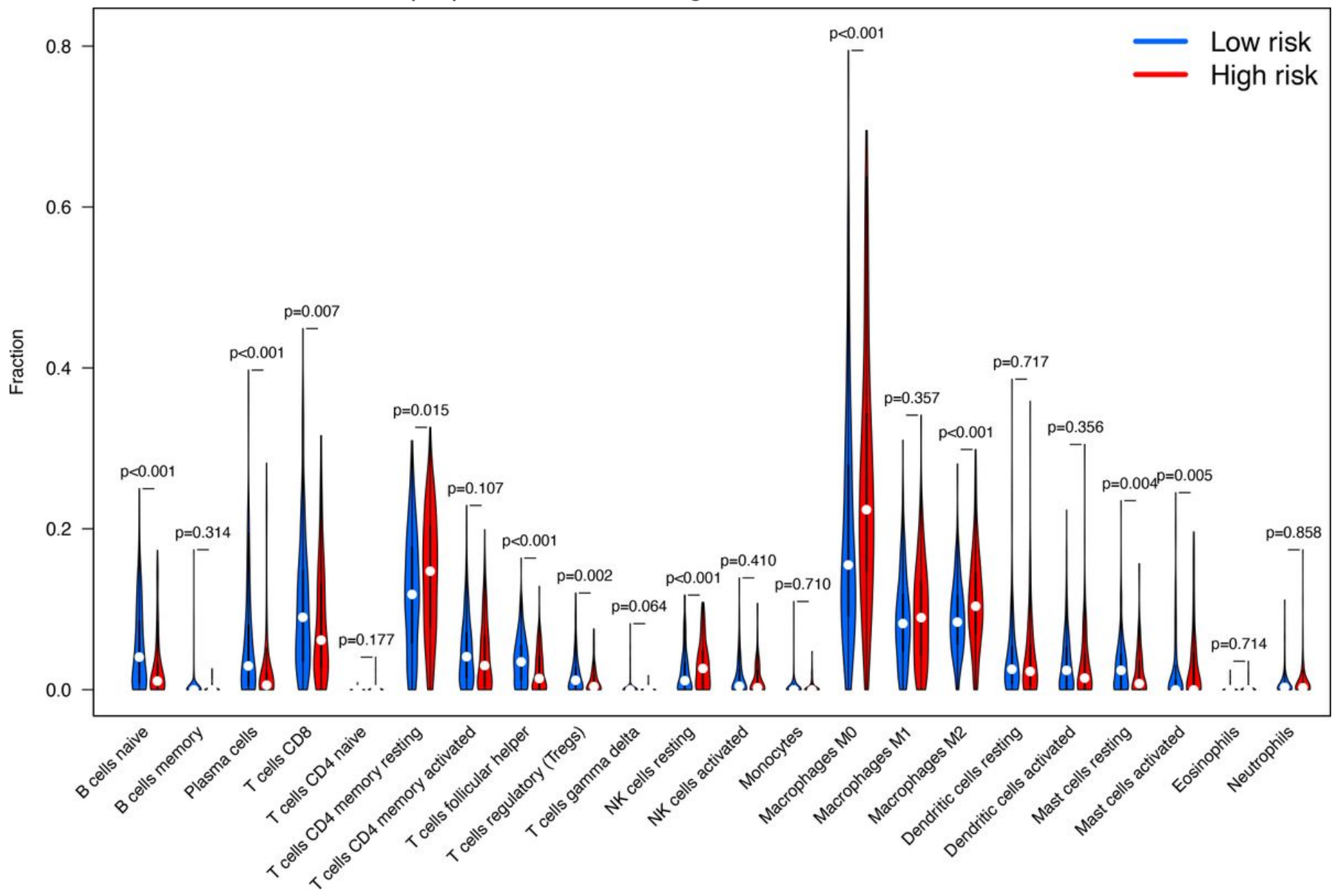

Figure 6

Estimation of tumor-infiltrating cells by the risk model 

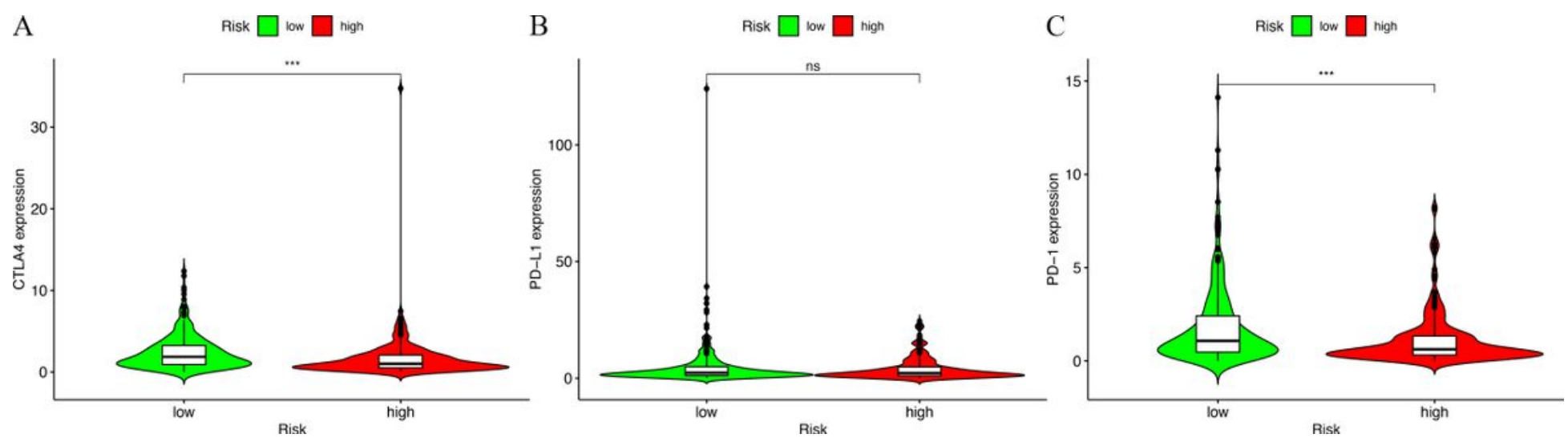

Figure 7

The gene expression of PD1, CTLA4 and PD-L1 in high-risk and low risk groups

A

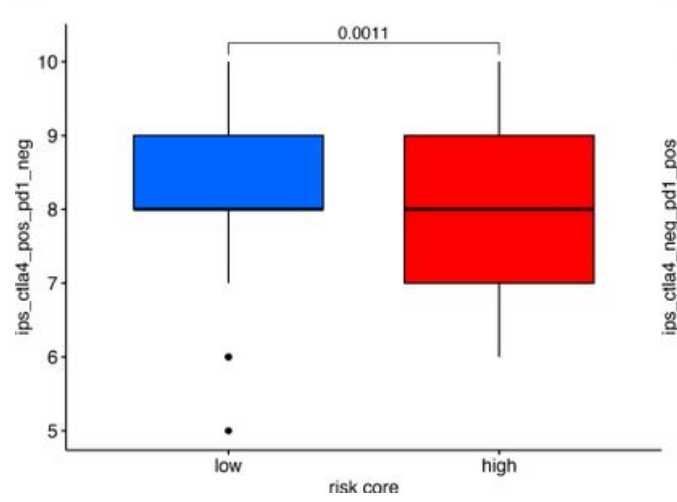

B

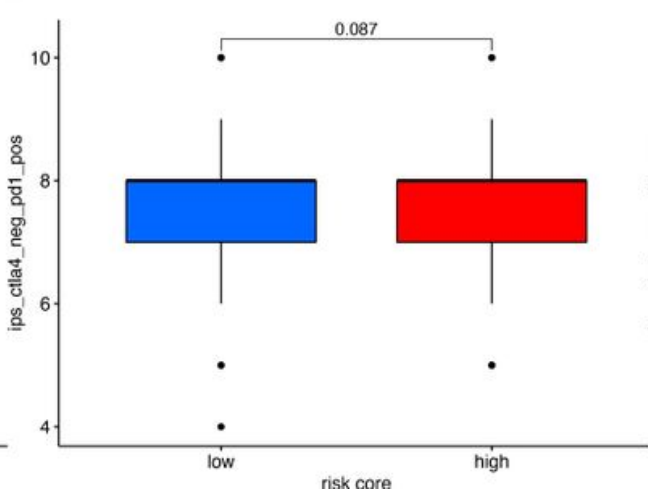

$\mathrm{C}$

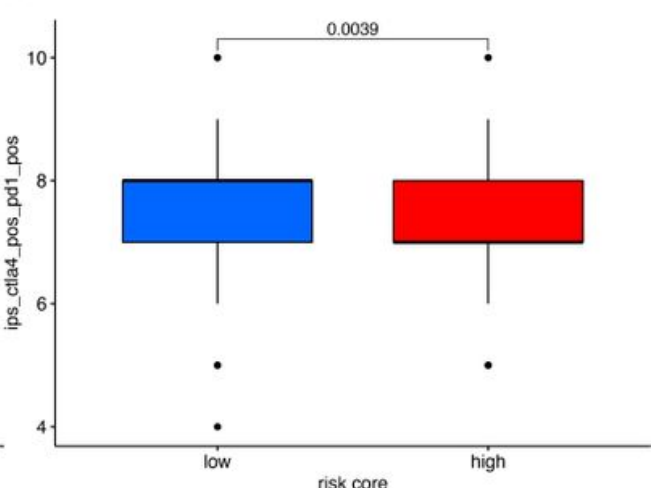

Figure 8

The association between the response to ICls and risk model in HNSCC 

A
Risk 追 low 官 high
B
Risk 追 low 官 high
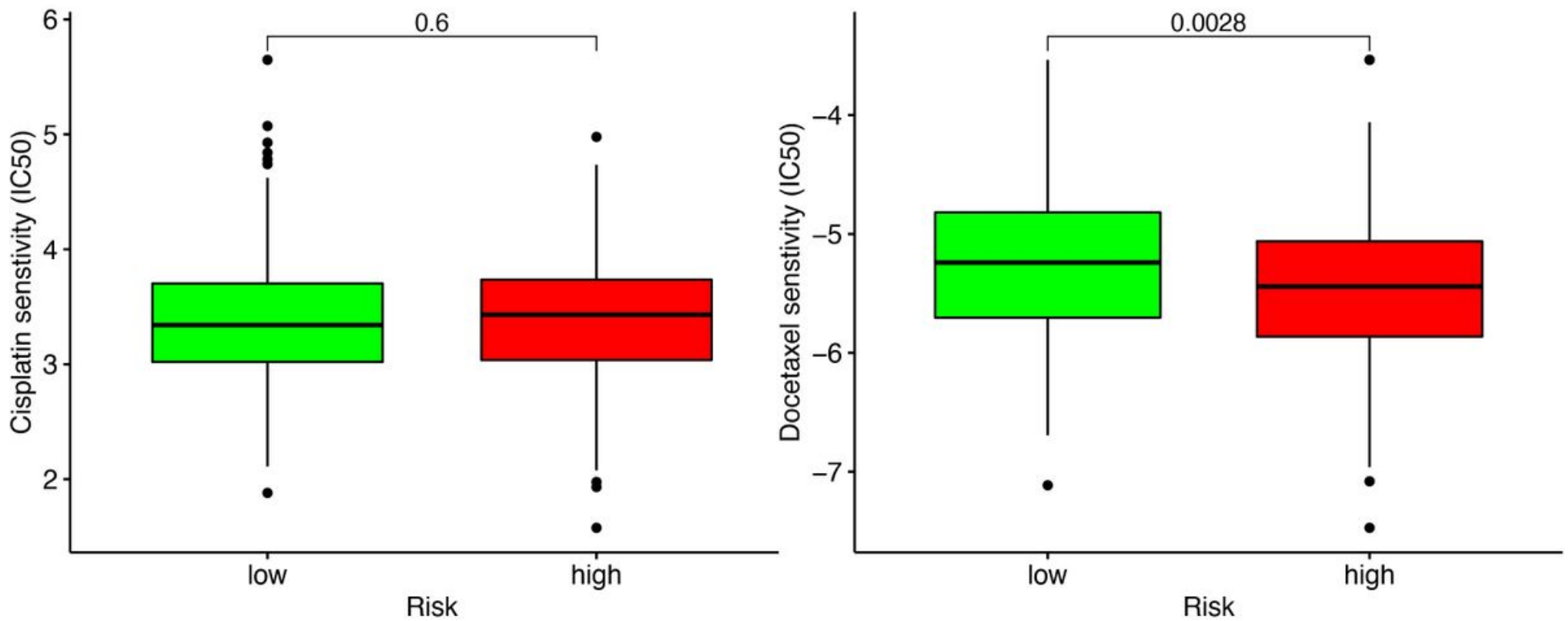

C

Risk 追 low 官 high

D

Risk 追 low 官 high
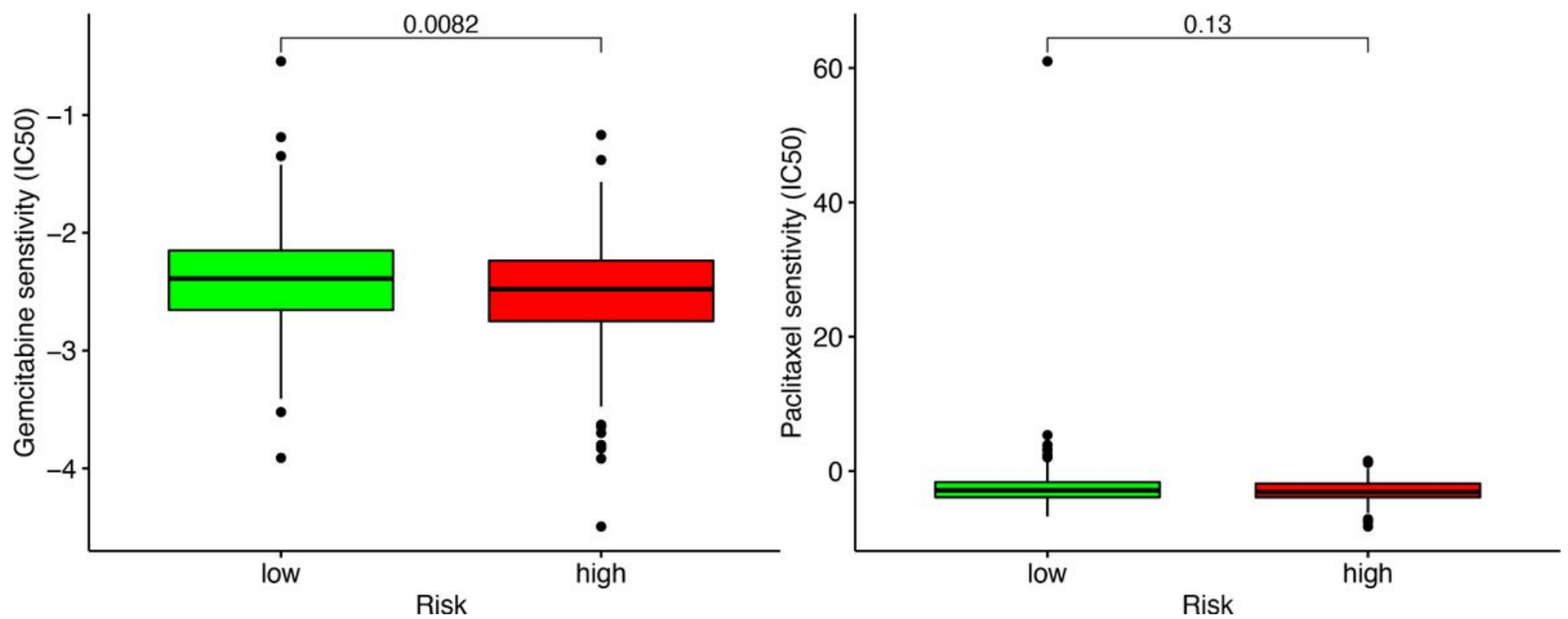

Figure 9

The associations between risk model and the efficacy of common chemotherapeutics (including cisplatin, docetaxel, gemcitabine and paclitaxel) in HNSCC

\section{Supplementary Files}

This is a list of supplementary files associated with this preprint. Click to download.

- FigureS1.pdf

- FigureS2.pdf 
- TableS1.pdf

Page 24/24 